\title{
Evaluation of Nutrient Foramina of the Dry Adult Human Femur Bone of North Indian Population
}

\author{
Ashok Kumar Sing ${ }^{1}$, Rekha Kumari ${ }^{2}$ \\ ${ }^{1}$ Associate Professor, Department of Anatomy, Vardhman Institute of Medical Science, pawapuri, Nalanda, Bihar, ${ }^{2}$ Assistant Professor, Department of \\ Anatomy, Vardhman Institute of Medical Science, pawapuri, Nalanda, Bihar.
}

\section{Abstract}

Introduction: The Femur bone is a highly vascular structure with unique features in its blood supply via numerous foramina located over its different segments, being named as vascular foramina. Among vascular foramina, nutrient foramen is an important one which gives way to the nutrient artery. Aim: To evaluate the nutrient foramina of the dry adult human femur bone of north Indian population. Subjects and Methods: The present study was undertaken using 50 dry adult human Femur bones of North Indian population. The samples were collected from the Anatomy department of the institute. Results: In the present study, among foramina of 50 dry adult human Femora, 31 Femora had single nutrient foramen, 18 had two nutrient foramina and 1 had three nutrient foramina. The medial lip of linea aspera of Femur depicted the presence of nutrient foramina in majority of bones suggesting the entry zone for nourishment of shaft by perforating arteries. Size of nutrient foramina were ranging from size $\geq 0.55 \mathrm{~mm}$ to size $\geq 1.27 \mathrm{~mm}$. Direction of nutrient foramina of all the Femora were directed upwards. Conclusion: This study may help orthopaedic surgeons in planning the surgical treatment of fracture of Femur with a possible reduction in post-operative complications. Combined periosteal and medullary blood supply to the bone cortex helps to explain the success of intramedullary reaming of long bone fractures particularly in the weight bearing Femur.

Keywords: Femur, Nutrient foramina, Ischaemic necrosis, Perforating arteries, Nutrient artery.

Corresponding Author: Dr. Ashok Kumar Singh, Associate Professor, Department of Anatomy, Vardhman Institute of Medical Science, pawapuri, Nalanda, Bihar.

Received: September 2019

Accepted: October 2019

\section{Introduction}

The nutrient artery is the principal source of blood supply to the long bone and is particularly important during its active growth period in the embryo and fetus, as well as during the early phase of ossification. ${ }^{[1]}$ The nutrient foramen is defined as the largest foramen present on the shaft of long bones allowing nutrient artery to enter the bone, the role of which is important in providing nutrition and growth of long bones. Knowledge of location and number of nutrient foramina in long bones is therefore important in orthopaedic surgical procedures such as joint replacement surgeries, fracture fixation, bone grafts and vascularised bone microsurgery. ${ }^{[2]}$ Bones are structures that adapt to their mechanical environment and from a fetal age adapt to the presence of naturally occurring holes which allow blood vessels to pass through the bone cortex. $^{[3]}$

Gopalakrishna K and Rathna BS concluded that knowledge on incidence of the nutrient foramen will help the orthopaedic surgeon in avoiding vascular injury, to assess the prognosis of surgery and to get better result in bone graft and fracture healing and also has academic, clinical and medico legal importance. ${ }^{[4]}$ Krishna SM et al, stated that thorough understanding of nutrient foramina has crucial role in orthopaedic surgical procedures like bone resection and transplantation. ${ }^{[5]}$

There is genetic, racial and ethnic variation amongst the humans. Therefore, this study was undertaken to evaluate the nutrient foramina of the dry adult human femur bone of north Indian population.

\section{Subjects and Methods}

The study was conducted at Vardhman Institute of Medical Sciences, Pawapuri, Nalanda, Bihar using 50 fully ossified and dry adult human Femur bones of either sex. The study was approved by institutional research committee. The Femur bones were collected from the department of anatomy. Only Femur bones with good conditions were included in the study. Deformed, damaged bones, bones with callous formation and unossified bones were excluded from the study.

The following materials were used to calculate the parameters in this study:

1) Marker pens 


\section{Singh d Fumari; Eualuation of Nutrient Faramina of the Dry Adult Ftuman Femur Bane}

2) 18, 20, 22, 24 gauge hypodermic needles.

The dry adult human Femur bones were numbered using the marker pen from 1 to 50 numbers in order. To locate the nutrient foramina, the Femur bone was divided into different segments:

\section{Location (a):}

i) Medial lip of linea aspera

ii) Lateral lip of linea aspera

iii) Medial surface

iv) Upper posterior surface.

\section{Location (b):}

i) At the junction of upper and middle $1 / 3 \mathrm{rd}$

ii) At the junction of middle and lower $1 / 3 \mathrm{rd}$

iii) At the middle $1 / 2$

iv) Other locations.

Based on the hypodermic gauge needle number which was admitted by the nutrient foramina, the foramina were categorized into 4 groups.

a) Large sized nutrient foramina: The foramina which admitted 18 gauge needle were considered to be between $1.27 \mathrm{~mm}$ or more $[\geq 1.27 \mathrm{~mm}]$.

b) Medium sized nutrient foramina: The foramina which admitted 20 gauge needle were considered to be between $0.90 \mathrm{~mm}$ and $1.27 \mathrm{~mm}[\geq 0.90 \mathrm{~mm}$ to < $1.27 \mathrm{~mm}]$.

c) Small sized nutrient foramina: The foramina which admitted 22 gauge needle were considered to be between $0.71 \mathrm{~mm}$ and $0.90 \mathrm{~mm}[\geq 0.71 \mathrm{~mm}$ to $<0.90$ $\mathrm{mm}]$.

d) Very small sized nutrient foramina: The nutrient foramina which admitted 24 gauge needle were considered to be between $0.55 \mathrm{~mm}$ and $0.71 \mathrm{~mm}[\geq$ $0.55 \mathrm{~mm}$ to $<0.71 \mathrm{~mm}$ ].

The direction of the needle was noted as follows:

a) If the needle passes upwards, it was noted as upper direction.

b) If the needle passes downwards, it was noted as lower direction.

c) If the needle passes horizontally, it was noted as horizontal.

Data obtained were analyzed using Statistical Package for Social Sciences (SPSS) for windows version 10.00.

\section{Results}

The study included 50 fully ossified and dry adult human femur bone. The femur bone was studied for the number, location, size and direction of the nutrient foramina. The result of the study revealed:

Number/ density of nutrient foramina.

Among 50 Femora studied, 31 Femora had single nutrient foramen, 18 Femora had two nutrient foramina and 1 Femur had three nutrient foramina. [Figure 1]

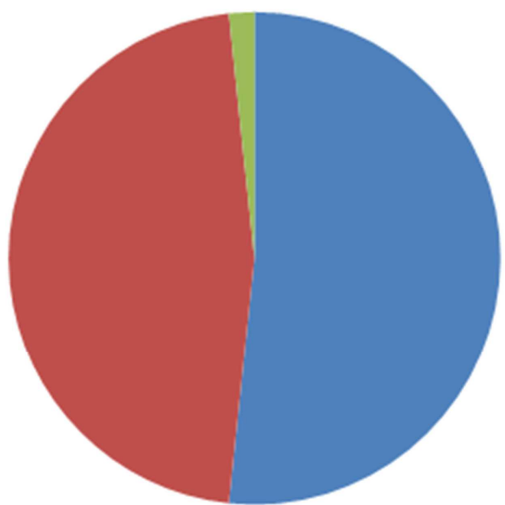

Figure 1: Number fo nutrient foramina

Location of nutrient foramina [Figure 2]

39 nutrient foramina were located at the junction of upper and middle $1 / 3$ rd of the Femur.

13 nutrient foramina were located at the junction of middle and lower $1 / 3$ rd of the Femur

16 nutrient foramina were located in middle half of the length of the Femur.

2 nutrient foramina were located in other locations of the Femur.

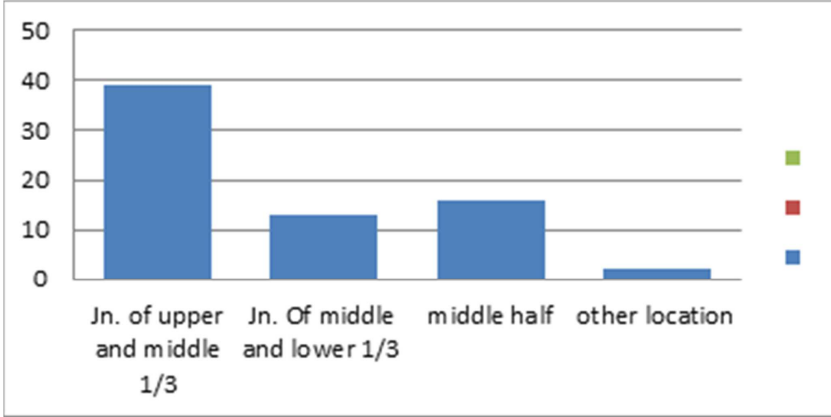

Figure 2: Location of nutrient foramina

Size of nutrient foramina [Figure 3]

5 nutrient foramina were large (of size $\geq 1.27 \mathrm{~mm}$ ).

45 nutrient foramina were medium (of size $\geq 0.90 \mathrm{~mm}$ to $<$ $1.27 \mathrm{~mm})$.

12 nutrient foramina were small (of size $\geq 0.71 \mathrm{~mm}$ to $<0.90$ $\mathrm{mm})$.

8 nutrient foramina were very small (of size $\geq 0.55 \mathrm{~mm}$ to $<$ $0.71 \mathrm{~mm})$.

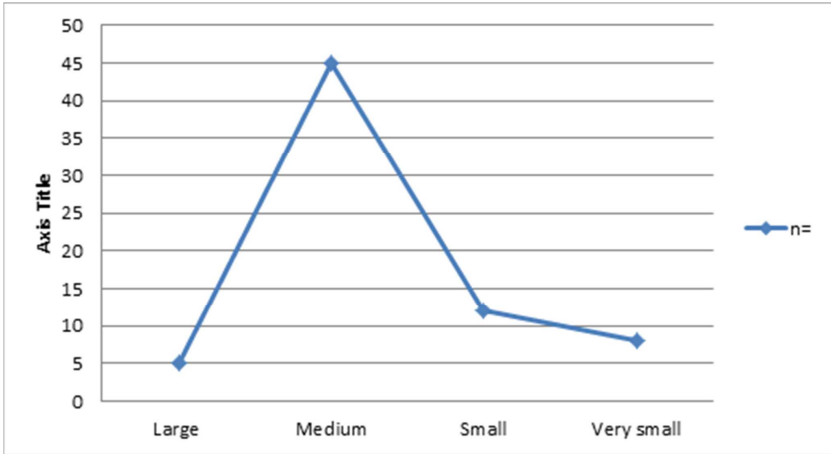

Figure 3: Size of nutrient foramina 


\section{Direction of nutrient foramina}

Among 50 Femora studied, nutrient foramina of all the Femora were directed upwards.

\section{Discussion}

A typical long bone is fed by four groups of arterial systems, which are - a nutrient artery, epiphyseal, diaphyseal and periosteal arteries. ${ }^{[6]}$ The Femur bone is a highly vascular structure with unique features in its blood supply. ${ }^{[7]}$ The nutrient arteries of the Femur usually arose from the perforating branches of the profunda femori artery, less commonly direct from the profunda femori and rarely from the lower segment of the femoral artery. The course of the nutrient arteries will be constant, the arteries run under the adductor magnus muscle, and through the nutrient foramina which lay on or near the linea aspera of the Femur. The nutrient foramina of the Femur are usually situated on the posterior surface, near the linea aspera of the Femur. ${ }^{[6]}$

Laing PG investigated the details of blood supply of the Femoral shaft in ten adults and seventeen newborn subjects. It was observed that a considerable number of Femora have only one main nutrient vessel supplying the shaft, even including the bones with two such vessels. No major artery entered the lower third of the shaft. ${ }^{[8]}$

Mysorekar VR studied for the number, position, symmetry of the diaphyseal nutrient foramina. His study showed that the arrangement of the diaphyseal nutrient foramina in the long bones of the limbs usually follows a definite pattern. There are often two nutrient foramina in the Femur. In the Femur, the nutrient foramina are restricted to the linea aspera or its immediate neighbourhood in the middle third of the bone. ${ }^{[9]}$ Henderson RG determined the positions of the Femoral and Tibial nutrient foramina by direct measurement in rats of 40, 49 and 59 days of age using a travelling microscope. The Femoral nutrient foramina remained constant in position with increasing age. In case of the Femur, this can be accounted for entirely by differences in growth ratesat the epiphyseal plates of the femur compensating for the disproportion in the distances of the foramen from the two plates. $^{[10]}$

Erika Collipal investigated the 140 dry femora for the location and number of diaphysis foramina. The nutrient foramen of the Femur was located in the linea aspera in the $72.5 \%$ and in the $21.25 \%$ in the medial surface and in the $6.25 \%$ in the lateral surface of the diaphysis of the bone. This data was used as reference for surgical procedures of the lower limb. ${ }^{[1]}$

Pereira GAM studies showed that the location and the number of nutrient foramina in the diaphysis of 885 long bones of the upper and lower limbs of adults. The location of the nutrient foramina is predominant on the posterior aspect of the lower limb long bones. The majority of the bones studied had a single nutrient foramen, which may represent a single source of blood supply. This data was used for comparison and in surgical procedures and in the interpretation of radiological images. ${ }^{[12]}$
In the present study, the nutrient foramina of 50 dry adult human Femora, 31 Femora had single nutrient foramen, 18 had two nutrient foramina and 1 had three nutrient foramina. Location of 39 nutrient foramina were located at the junction of upper and middle $1 / 3$ rd of the femur, 13 nutrient foramina were located at the junction of middle and lower 1/3rd of the femur, 16 nutrient foramina were located in middle half of the length of the femur, 2 nutrient foramina were located in other locations of the femur bones. Size of nutrient foramina were ranging from size $\geq 0.55 \mathrm{~mm}$ to size $\geq 1.27 \mathrm{~mm}$. Direction of nutrient foramina of all the Femora were directed upwards and density of nutrient foramina was found more close to medial lip of linea aspera and at the junction of upper and middle 1/3rd of the shaft of Femur.

Blood supply to Femur bone is essential during the growing period, during the early phases of ossification, and in surgical procedures such as bone grafts, tumour resections, traumas, congenital pseudoarthrosis, and in transplant techniques in orthopaedics.

\section{Conclusion}

The nutrient foramen emphasizes the significance of perforating arteries to cater the blood supply to medullary wall and bone marrow. The nutrient artery zone of the Femur has to be tackled with utmost caution during surgical interventions for subtrochanteric fractures as otherwise it may lead to severe haemorrhage. The present study conducted on nutrient foramina of 50 dry adult human Femora arrived at a conclusion that the density of nutrient foramina was found more close to medial lip of linea aspera and at the junction of upper and middle 1/3rd of the shaft of femur. This location is highlighting, as the nutrient artery may be involved in iatrogenic (or) traumatic injuries. With the results of study of these foramina, it is possible to arrive at surgical implications of the fractures of the Femur like avoiding the entry zones of arteries during plating procedures etc. This study may help orthopaedic surgeons in planning the surgical treatment of fracture of Femur with a possible reduction in post-operative complications. Combined periosteal and medullary blood supply to the bone cortex helps to explain the success of intramedullary reaming of long bone fractures particularly in the weight bearing Femur.

\section{References}

1. Lewis OJ. The blood supply of developing long bones with special reference to the metaphyses. J BoneJoint Surg 1956;38:928-33.

2. Rao VS, Kothapalli J. The diaphyseal nutrient foramina architecture- a study on the human upperand lower limb long bones. Int J Pharm Bio Sci 2014;9(1):36-41.

3. Gotzen N, Cross AR, Ifju PG, Rapoff AJ. Understanding stress concentration about a nutrient foramen.J Biomech 2003;36(10):1511-21.

4. Gopalakrishna K, Rathna BS. The study on the incidence and direction of nutrient foramina in the diaphysis of femur bone of South Indian origin and their clinical importance. Int $\mathrm{J}$ Basic Life Sci 2014;2(2):11-19.

5. Krishna SM, Kumar PU, Sirisha V, Rajesh V. Morphologic and 


\section{Singh \& Kumari; Evaluatian of Nutrient Faramina of the Dry Adult Ftuman Femur Bane}

morphometric study of the nutrient foramina in dry human femur bones of Telangana region. Int J Anat Res 2016;4(2):246468.

6. Datta AK. Principles of general Anatomy. 6th ed. Kolkata, India: Current books internat ional; 2010.p.75-77.

7. Laroche M. Intraosseous circulation from physiology to disease. Joint Bone Spine. 2002; 69: 262-69.

8. Laing PG. The blood supply of the femoral shaft; an anatomical study. J Bone Joint Surg Br. 1953 Aug; 35-B (3): 462-6.

9. Mysorekar VR. Diaphysial nutrient foramina in human long bones. J Anat. 1967 September; 101(4): 813-822.
10. Henderson RG. The position of the nutrient foramen in the growing tibia and femur of the rat. J Anat. 1978 Mar; 125(3):593-9.

11. Erika Collipal, Ramiro Vargas, Ximena Parra, Héctor Silva, Mariano del Sol. Diaphyseal Nutrient Foramina in the Femur, Tibia and Fibula Bones. Int J. MorphoL. 2007; 25(2):305-308.

12. Pereira G A M, Lopes P T C, Santos A M P V \& Silveira F H S. Nutrient foramina in the upper and lower limb long bones: Morphometric study in bones of Southern Brazilian adults. Int. J. Morphol 2011; 29(2):514-520.

Copyright: ( $\odot$ the author(s), publisher. Academia Anatomica International is an Official Publication of "Society for Health Care \& Research Development". It is an open-access article distributed under the terms of the Creative Commons Attribution Non-Commercial License, which permits unrestricted non-commercial use, distribution, and reproduction in any medium, provided the original work is properly cited.

How to cite this article: Singh AK, Kumari R. Evaluation of Nutrient Foramina of the Dry Adult Human Femur Bone of North Indian Population. Acad. Anat. Int. 2019;5(2):18-21.

DOI: dx.doi.org/10.21276/aanat.2019.5.2.6

Source of Support: Nil, Conflict of Interest: None declared. 